\title{
Coherent suppression of backscattering in optical microresonators
}

\author{
Andreas $\varnothing$. Svela ${ }^{1,2,3}$, Jonathan M. Silver $\mathbb{B}^{1,4}$, Leonardo Del Bino ${ }^{1,5,6}$, Shuangyou Zhang ${ }^{1,5}$, Michael T. M. Woodley ${ }^{1,2,6}$, \\ Michael R. Vanner, ${ }^{2,3}$ and Pascal Del'Haye (i) ${ }^{1,5,7}$
}

\begin{abstract}
As light propagates along a waveguide, a fraction of the field can be reflected by Rayleigh scatterers. In high-qualityfactor whispering-gallery-mode microresonators, this intrinsic backscattering is primarily caused by either surface or bulk material imperfections. For several types of microresonator-based experiments and applications, minimal backscattering in the cavity is of critical importance, and thus, the ability to suppress backscattering is essential. We demonstrate that the introduction of an additional scatterer into the near field of a high-quality-factor microresonator can coherently suppress the amount of backscattering in the microresonator by more than $30 \mathrm{~dB}$. The method relies on controlling the scatterer position such that the intrinsic and scatterer-induced backpropagating fields destructively interfere. This technique is useful in microresonator applications where backscattering is currently limiting the performance of devices, such as ring-laser gyroscopes and dual frequency combs, which both suffer from injection locking. Moreover, these findings are of interest for integrated photonic circuits in which back reflections could negatively impact the stability of laser sources or other components.
\end{abstract}

\section{Introduction}

Optical whispering-gallery-mode (WGM) microresonators are widely used in photonics for a range of applications, including sensing and metrology ${ }^{1-6}$, optomechanics $^{7-9}$, quantum optics ${ }^{10,11}$, and classical and quantum information processing ${ }^{12-14}$. Microresonators can adopt a range of different geometries, but for all of them, imperfections in the resonator surface or bulk material can cause scattering of some portion of the light into the counter-propagating whispering-gallery mode ${ }^{15-18}$.

Backscattered light in microresonators can limit the application performance, for example, causing unwanted injection locking in laser gyroscopes operating at low rotational speeds ${ }^{19-21}$ or in dual frequency $\operatorname{combs}^{22,23}$. Backscattering also reduces the nonlinear enhancement

\footnotetext{
Correspondence: Andreas Ø. Svela (asvela@ic.ac.uk) or Pascal Del'Haye (pascal.delhaye@mpl.mpg.de)

${ }^{1}$ National Physical Laboratory, Teddington TW11 OLW, UK

${ }^{2}$ Blackett Laboratory, Imperial College London, London SW7 2AZ, UK

Full list of author information is available at the end of the article
}

and contributes to back reflections in devices relying on symmetry breaking of counter-propagating fields ${ }^{24}$ for sensing $^{25}$, optical computing ${ }^{26,27}$ or isolator ${ }^{28}$ applications. Furthermore, control over backscattering permits tuning of the standing wave pattern to maximise coupling by moving an anti-node of the standing wave along the resonator perimeter, which is beneficial for systems relying on evanescent coupling, such as evanescent optomechanics $^{8}$, or biomedical near-field sensors ${ }^{29}$. In addition, telecom applications ${ }^{14,30}$ can benefit from lower backscattering levels.

The imperfections causing backscattering in microresonators are typically distributed around the cavity but can be approximated as a single scatterer with specific amplitude and phase, as the coherence length of the circulating field is much longer than the cavity round-trip length ${ }^{31}$. The reflected field is resonant in the cavity and therefore builds up in the counter-propagating direction ${ }^{32,33}$. This coupling of the two travelling-wave modes generally results in nonorthogonal chiral eigenmodes composed of unequal superpositions of the two travelling-wave modes $^{34}$. These 
superposition modes typically have different frequencies and losses. For high levels of backscattering, the mode splitting may be spectrally resolvable, i.e., detected as two separate resonances ${ }^{16,18}$. Previous experimental work has focused on controlling and changing the backscattering in such systems with intrinsically high backscattering rates, tuning the mode splitting with a near-field scatterer ${ }^{35,36}$ or inducing chirality for light flow control $^{37,38}$, without investigating backscattering suppression. The backscattering problem is now attracting interest in the community, and recently, an optomechanical method to reduce backscattering was demonstrated $^{39}$, showing suppression from resolved to unresolved mode splitting.

Here, we show an unprecedented $34 \mathrm{~dB}$ suppression of the backscattered light from a WGM resonator, limited by the photodetector noise. This is achieved by manipulating the position of a sub-wavelength-size scatterer within the near field of the optical mode (Fig. 1a), coherently controlling the effective backscattering. We demonstrate the effect in two silica rod microresonators with intrinsically low backscattering, meaning neither one shows resolved frequency mode splitting (resonator diameters $d=2.7 \mathrm{~mm}, 1.7 \mathrm{~mm}$ and $\left.Q \simeq 2 \times 10^{8}, 1.1 \times 10^{9}\right)$.

In our setup, a sub-wavelength tungsten tip (Fig. 1b-d) controls the backscattering by coherently scattering additional light from the pumped optical mode into the counter-propagating mode (Fig. 1e), leading to interference between the intrinsic backscattering and that caused by the metal tip ${ }^{34}$. With sufficient induced backscattering and an appropriate phase offset between the intrinsic and induced backscattered waves, the net backscattering can be made to vanish. As the radial position of the tip controls the amount of induced backscattering and the azimuthal position governs the phase offset between the intrinsic and induced backscattered waves, the tip position can coherently control the net backscattered field (Fig. 1f). When the tip induces backscattering of equal magnitude to the intrinsic backscattering, we call this critical tip coupling. The tip also scatters to free-space modes; however, for a small tip
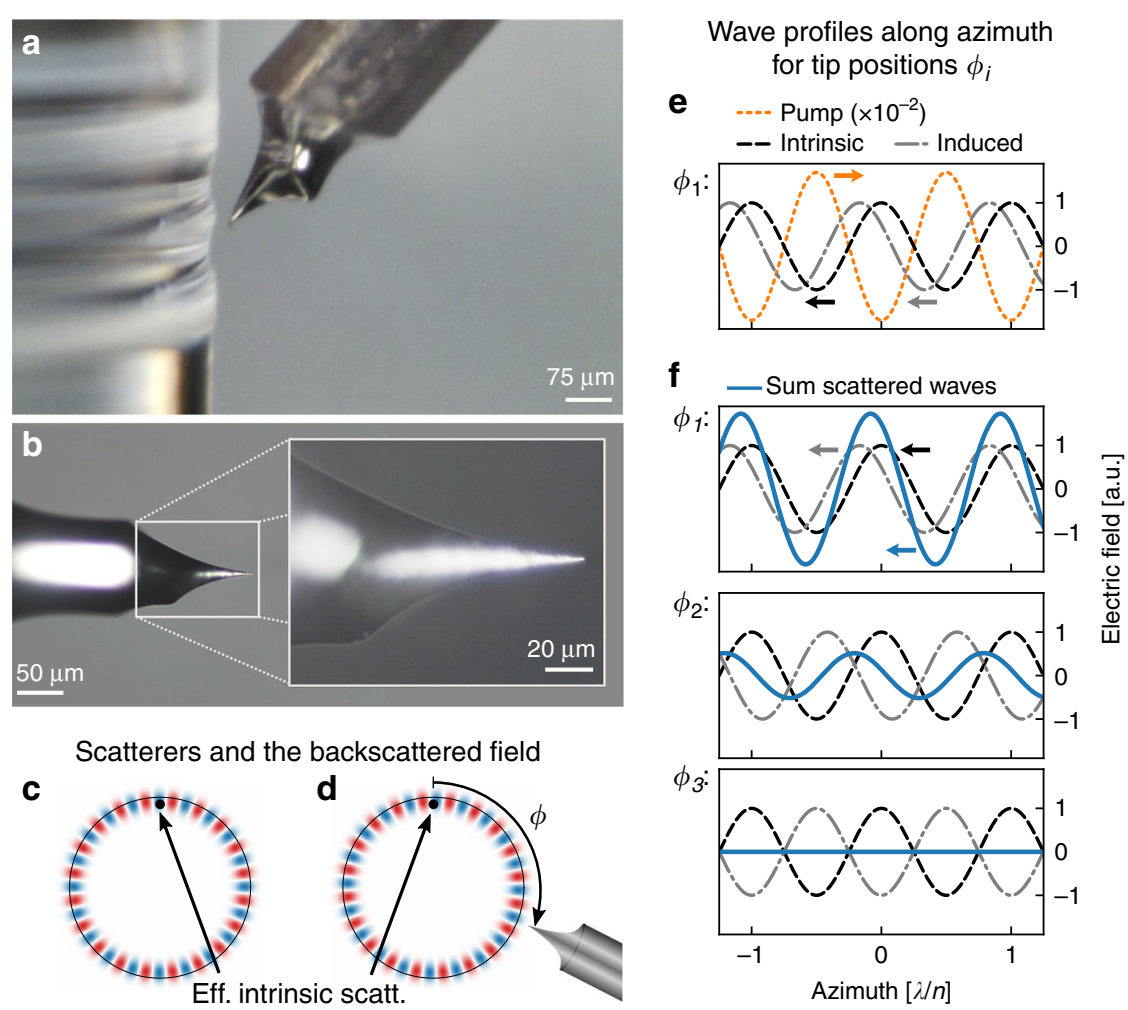

Fig. 1 Principle of backscattering control. $\mathbf{a}-\mathbf{b}$ Micrographs of the microresonator and tungsten tip. $\mathbf{c}$ Illustration of the real part of the backscattered field (cavity size and wavelength not to scale); the scatterer is represented by one effective scatterer here (black dot). $\mathbf{d}$ Introducing a second scatterer at an azimuthal angular distance $\phi$ from the effective scatterer, the backscattered field changes depending on $\phi$. e Illustration of wave profiles along the azimuth showing the counter-clockwise-propagating backscattered waves due to the clockwise-propagating pump field. The backscattered field amplitudes are small compared to the pump, as only a small fraction of the light is backscattered. The tip is critically coupled (equal amplitudes for intrinsic and induced backscattered waves). f Backscattered waves and their sum under critical coupling (equal amplitudes) for different azimuthal positions $\phi_{i}$ of the tip, corresponding to phase offsets between the effective intrinsic scatterer and the induced scatterer $(2 \mathrm{~m}+q)$ $\pi$ for integer $m$ and $q=1 / 3,5 / 6$ and 1, showing both constructive and destructive interference 
diameter, the reduction in the Q-factor is small. This technique enables full control of the amplitude and phase of the effective backscattering in a microresonator, and in this experiment, we show that it can be reduced by orders of magnitude beyond the unresolved frequency splitting level.

\section{Results}

Response of the resonator to a perturbation in the near field

We studied the backscattering amplitude and resonance linewidth as functions of the distance of the tungsten tip from the resonator surface $r$ and its azimuthal position $\phi$ while keeping the tip in the resonator plane ( $x y$ plane in Fig. 2a). The microrod resonator was pumped with a tuneable, $1.55 \mu \mathrm{m}$ continuous-wave laser using a tapered optical fibre ${ }^{40}$ to couple light into the cavity. The $20 \mathrm{~mW}$ input optical power was scanned downwards in frequency to obtain spectral data of the resonance. To avoid thermal broadening ${ }^{41}$ of the resonance, the laser frequency was scanned at a rate of $450 \mathrm{GHz} \mathrm{s}^{-1}$. The nonlinear Kerr effect is faster than the scanning rate and thus causes some broadening; however, with low input power, this broadening is small. A circulator allowed the backscattered light from the cavity to be monitored with a photodetector, along with the cavity transmission

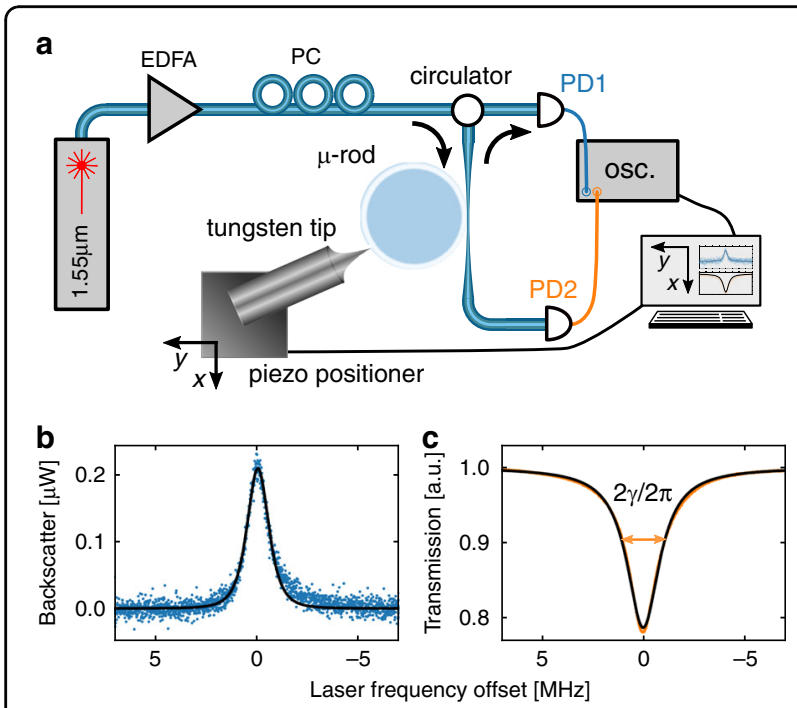

Fig. 2 Experimental setup and example measurements. a Optical circuitry, consisting of a fibre-coupled external cavity diode laser $(1.55 \mu \mathrm{m})$ amplified by an erbium-doped fibre amplifier (EDFA), a polarisation controller (PC) to optimise the coupling to the desired resonator mode, a circulator to separate the propagation directions in the fibre, and photodetectors to monitor the backscattering (PD1) and transmission of the microresonator (PD2). The tungsten tip near-field probe was fixed to a piezo positioner. Example backscattering $\mathbf{b}$ and transmission c spectra with fitted Lorentzians (black) for one position of the near-field probe, showing the transmission linewidth $2 \gamma$ (see Fig. 2a). The field-perturbing probe was fixed to a computer-controlled piezoelectric positioner for threeaxis control of the tip position.

The tip position was raster scanned with a step size of $50 \mathrm{~nm}$ over a grid of $(x, y)$ positions in the resonator plane. At each position, transmission and backscattering spectra were simultaneously recorded. The spectra were subsequently fitted with Lorentzian functions (see "Materials and methods" section), as shown for the examples in Fig. 2b, c, to extract the backscattering Lorentzian amplitude $A_{\mathrm{b}}$ and the pump resonance half-linewidth $\gamma$, respectively. The resulting data grids for the resonator with $Q \simeq 2 \times$ $10^{8}$ are shown in Fig. 3a, b, where each pixel corresponds to a position on the measurement grid. For positions corresponding to $r<0$, the tip was touching the resonator and sliding along its surface due to the force applied by the piezo positioner.

We performed numerical fitting of the linewidth and amplitude data vs. the two spatial coordinates to determine the position of the resonator surface and radial distance dependences and estimate the periodicity in the fringe pattern and suppression achieved for the backscattering amplitude.

\section{The near-field decay and resonator boundary}

Assuming a linear coupling between the evanescent near field and the tip, we expect the radial dependence of the linewidth to have the same functional dependence as the energy density in the near field. The evanescent electric field from a waveguide decays exponentially with the perpendicular distance from the surface-i.e., for a WGM resonator, with the radial distance from the surface, $r$. The evanescent field can be expressed as $E_{\text {ev }}(r)=E_{\text {surf }} \exp (-\alpha r)$, where $E_{\text {surf }}$ is the field strength at the surface, and the decay length is

$$
\alpha^{-1}=\frac{\lambda}{2 \pi \sqrt{n^{2}-1}}
$$

for a field of vacuum wavelength $\lambda$ in a waveguide of refractive index $n$ surrounded by air ${ }^{42}$. With the evanescent field energy density proportional to $\left|E_{\mathrm{ev}}(r)\right|^{2} \propto \exp (-2 \alpha r)$, we expect the tip-induced loss and backscattering amplitude to also be proportional to this quantity.

However, the prefactors corresponding to coupling back to the resonator clockwise and counter-clockwise directions, coupling to free-space modes, and absorption by the tip are dependent on the size, geometry, and material of the scatterer, as well as on the polarisation of the mode. As propagating modes of different orders than the mode in question have different resonance frequencies, we do not expect light to couple to other WGM modes. Note that in addition to coupling into free space modes, the tip could also induce losses via coupling into non-guided 

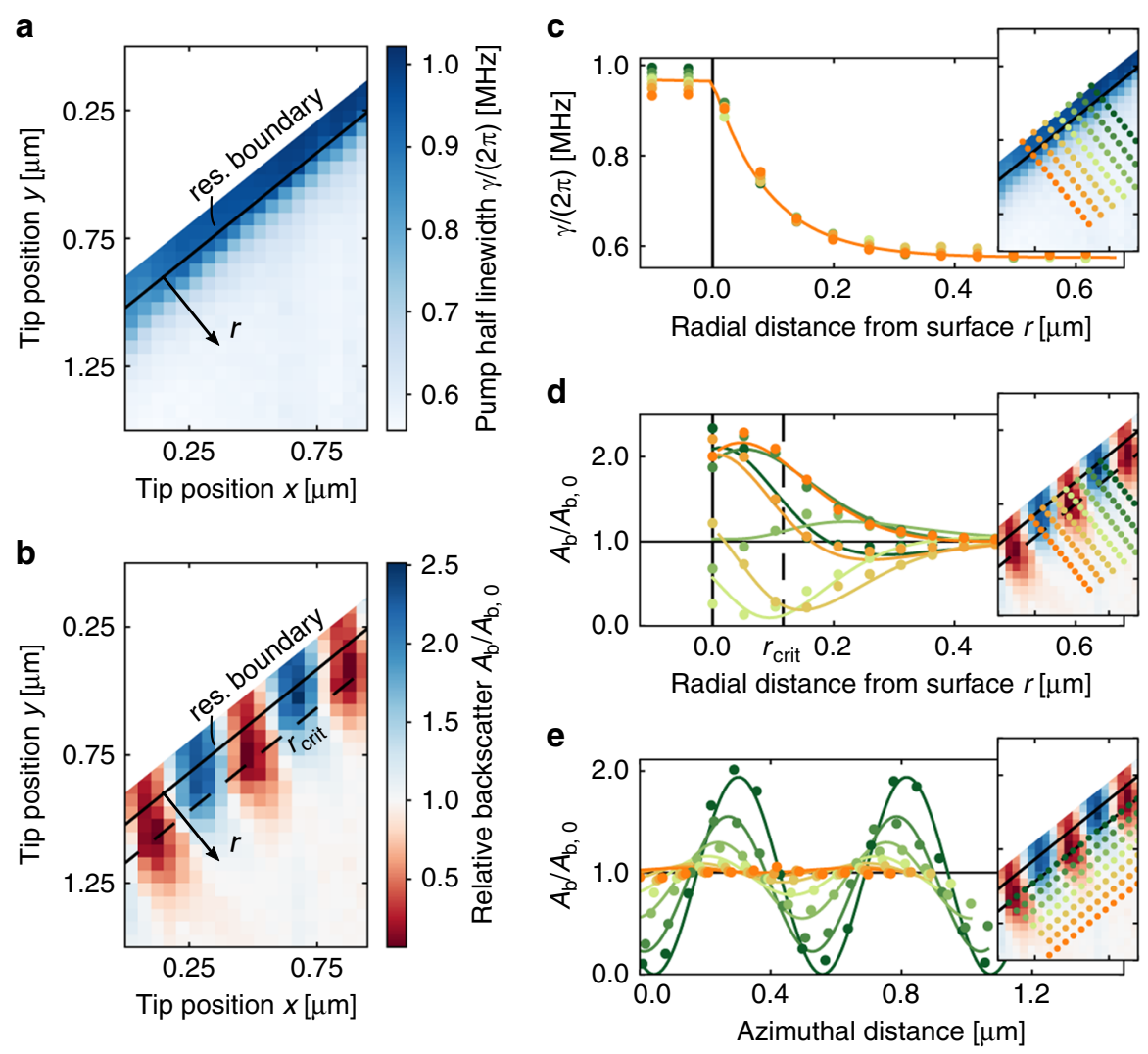

Fig. 3 Resonator response to the tungsten tip in the near field. a Total linewidth (and equivalently Q-factor) and $\mathbf{b}$ backscattering amplitude for tip positions in the resonator plane, normalised by the intrinsic backscattering amplitude $A_{b, 0}$. The black lines $(r=0)$ indicate the fitted resonator surface-measurements shown as $r<0$ were obtained while the tip was touching the resonator surface, resulting in the tip sliding along the surface. Maximum backscattering suppression is found along the dashed line (critical tip coupling); for $r<r_{\text {crit, }}$ the tip is over-coupled, reducing the suppression. c-e Fits (lines) and interpolated data (circles) along cross sections through the experimental data in panels a and $b$. The insets show where the cross sections were taken. $\mathbf{c}$ Radial tip position dependence of the linewidth and $\mathbf{d}$ backscattering. $\mathbf{e}$ Tip azimuthal position dependence of the backscattering

modes within the fused silica. It has previously been shown that for a silica sub-wavelength tip, it is the tip size relative to the mode volume that determines the amount of induced losses ${ }^{35}$, and furthermore, the tip can cause mode splitting with no change in the quality factor compared to when the tip is not present ${ }^{34,43}$.

The linewidth data were fitted with a piecewise function comprising an exponential decay from the resonator surface and a linear plateau for the tip positions $r<0$ where the tip is touching the resonator surface (detailed fitting function given in "Materials and methods" section). The fitted interface between the plateau and the exponential decay determines the resonator surface $r=0$, shown as black lines in Fig. 3a, b. Figure 3c shows cross sections of interpolated values (circles) and the fit (solid) to the linewidth data along the radial direction. The fit gives an exponential decay length for the linewidth of $\left(2 \alpha_{\gamma}\right)^{-1}=92 \mathrm{~nm}$, compared to the calculated near-field power decay length of $(2 \alpha)^{-1}=119 \mathrm{~nm}$ using Eq. (1) for silica, $n=1.44$ at $\lambda=1553 \mathrm{~nm}$. The steeper decay coefficient in the experimental data compared to the calculated value can be explained by the tip geometry; as the tip approaches the surface, the effective scattering cross section/polarisability increases, leading to an increasingly larger broadening.

\section{Backscattering suppression analysis}

The backscattering data in Fig. 3b show maxima and minima when the tip is some distance from the resonator rather than at the surface owing to over-coupling of the tip -i.e., the induced backscattering is larger than the intrinsic backscattering. The radial distance at which the tip minimises the backscattering, $r_{\text {crit }}$, is indicated. The data $r \geq 0$ are fitted with a function that is effectively an exponential decay of coefficient $2 \alpha_{\mathrm{b}}$ multiplied by a fringe pattern (see "Materials and methods" section). The fringe pattern arises due to the relative phase change between the intrinsic and induced backscattering as the tip 
is translated along the azimuthal direction. The expected periodicity of the fringe pattern can be estimated by $\lambda / 2 n=539 \mathrm{~nm}$ for silica at our pump wavelength (see Supplementary information). Figure $3 \mathrm{~d}$, e show cross sections of interpolated values (circles) and the fit (solid) along the radial and azimuthal directions, respectively, for the backscattering data. The fit gives a fringe period of $515 \mathrm{~nm}$ and a decay coefficient for the backscattering of $\left(2 \alpha_{\mathrm{b}}\right)^{-1}=99 \mathrm{~nm}$, similar to the linewidth decay length. The current setup is stable enough for the fringe pattern position to remain in place for measurements of at least $45 \mathrm{~min}$.

\section{Backscattering suppression in resonators with higher Q-factors}

Figure 4 shows data from a similar measurement for a resonator with $Q \simeq 1.1 \times 10^{9}$. Due to the extremely high Q-factor, this measurement was performed with a lower scanning speed $\left(10 \mathrm{GHz} \mathrm{s}^{-1}\right)$ to avoid ring-down signals, calling for a lower input power $(\sim 40 \mu \mathrm{W})$ to avoid thermal broadening. The backscattering pattern now shows a decrease in the backscattering for all azimuthal positions because the backscattering is linewidth dependent (see "Materials and methods" section), and the linewidth decreases more than for the resonator in Fig. 3. The maximum backscattering suppression obtained, $34 \mathrm{~dB}$, is limited by the photodetector noise.

The total Q-factor of the system was calculated from $Q=\omega_{\text {pump }} /(2 \gamma)$ for pump frequency $\omega_{\text {pump }}=2 \pi \times$ 193.1 GHz. Given the effective taper coupling $\eta$, the quality factor $Q_{0}$ of the resonator with the tip present can be estimated as $Q_{0}=2 Q /(1+\sqrt{1-\eta})$, shown for different radial positions of the tip in Fig. 4c. The maximum suppression occurs when $Q_{0}=2.5 \times 10^{8}$.

\section{Discussion}

Optical microresonators provide prospects for miniaturised sensing and communications systems; however, backscattering compromises the performance of some microresonator-based systems. We have demonstrated a method for coherently suppressing the intrinsic backscattering in an optical WGM microresonator, with the suppression exceeding $34 \mathrm{~dB}$ (limited by photodetectors) from a level where frequency splitting is not resolved. Suppression of backscattering opens opportunities for pure travelling-wave resonators, improving the performance in microresonator applications where backscattering is a limiting factor. These applications include symmetry-breaking-based sensing or non-reciprocal systems, optomechanics applications, laser gyroscopes and dual frequency combs; thus, backscattering suppression enables the development of high-accuracy, portable optical spectroscopy systems, gyroscopes and other sensors. The technique is of particular interest for on-chip
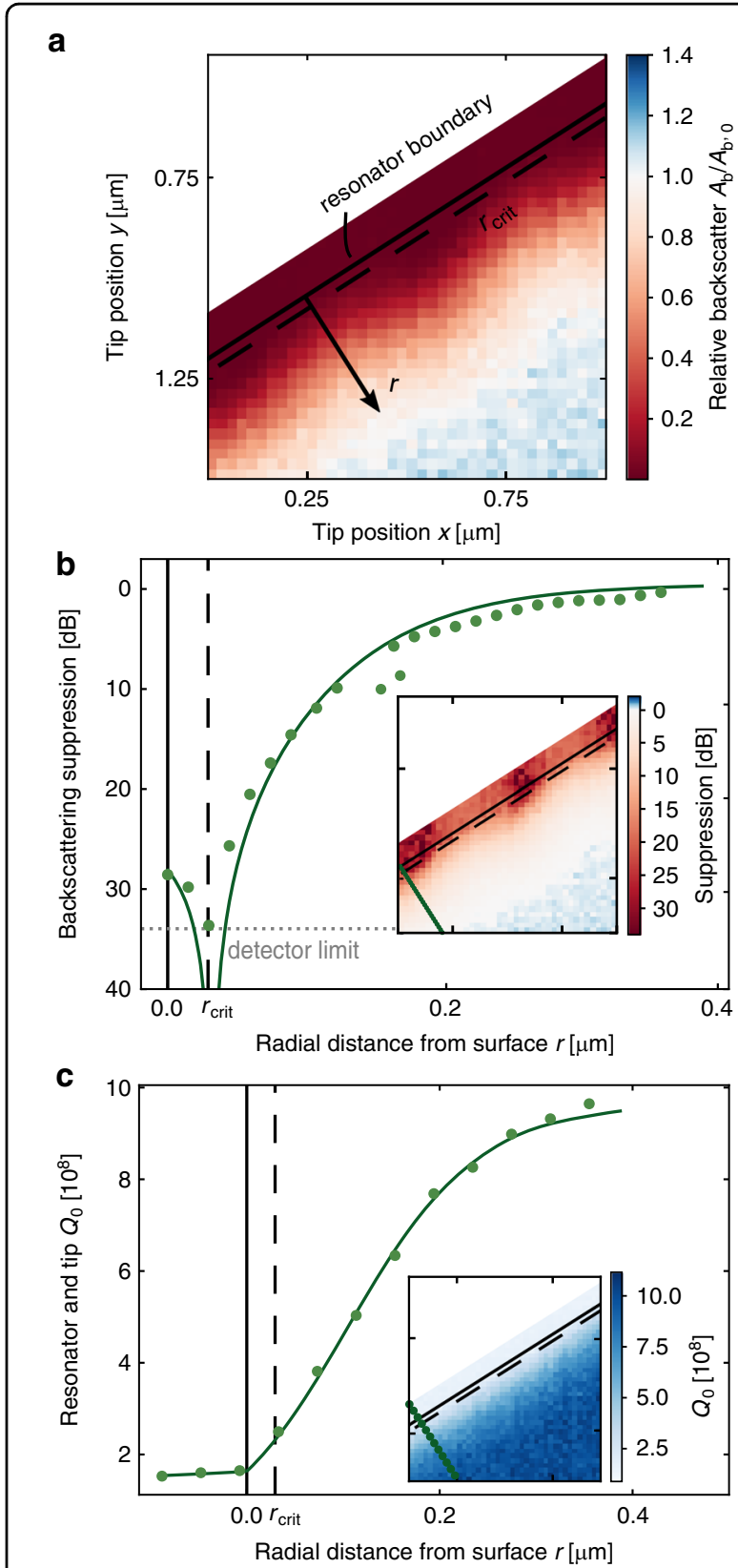

Fig. 4 Backscattering suppression and $Q_{0}$ in a high-Q resonator. a Backscattering amplitude measurement grid, with the resonator boundary and critical tip coupling line annotated. b Radial dependence of the backscattering suppression (circles, measurement; line, fit) along a radial line. The noise of the photodetector is indicated by the dotted grey line. Inset: suppression for all positions. The critical tip coupling at which the maximum suppression occurs is annotated. The measured suppression is limited by the photodetector noise. c Radial dependence of resonator and tip quality factor $Q_{0}$ along a radial line. Inset: $Q_{0}$ for all positions

resonators, where a scatterer can be permanently integrated on chip to coherently suppress back reflections. In addition, one could envision tuneable on-chip backscattering suppression with a MEMS-based device. 


\section{Materials and methods}

\section{Resonator and tapered fibre fabrication}

The rod resonators were machined using a $100 \mathrm{~W} \mathrm{CO}_{2}$ laser, milling commercially available 3-mm-diameter silica glass rods. The procedure followed that of Del'Haye et al. ${ }^{44}$, but to reach the highest Q-factor of $10^{9}$, the resonator was fabricated in a nitrogen atmosphere, seeking to avoid the formation of near-IR-absorbing $\mathrm{OH}$ groups. The rod was fixed to a spindle motor, and the glass was evaporated with the focused laser beam. First, the low (high) Q rod was milled down to a 2.7-mm-diameter (1.7-mm-diameter) cylindrical shape, and then, the resonator was created by making two ring cuts separated by $\sim 125 \mu \mathrm{m}(\sim 240 \mu \mathrm{m})$.

The tapered fibre was made from a stripped $125-\mu \mathrm{m}$ diameter standard single-mode silica optical fibre. The fibre was clamped to stepper motor stages, with a hydrogen flame placed under the fibre to heat it while simultaneously pulling symmetrically from both sides.

\section{Tungsten tip fabrication}

The fabrication of the tungsten tip was based on methods used in scanning tunnelling electron microscopy and atomic force microscopy tip fabrication ${ }^{45,46}$. The process relies on surface tension to form a meniscus of a solution around a piece of tungsten wire and on the aqueous electrochemical reaction

$$
\mathrm{W}(\text { solid })+2 \mathrm{OH}^{-}+2 \mathrm{H}_{2} \mathrm{O} \rightarrow \mathrm{WO}_{4}^{2-}+3 \mathrm{H}_{2} \text { (gas) }
$$

etching the solid tungsten (W) anode through oxidation. When the minimum potential difference $(1.43 \mathrm{~V})$ is overcome, the etching rate varies along the wire due to a hydroxide concentration gradient: the etching rate is slower at the top of the meniscus because the 'hydroxide supply' is lower in the meniscus above the horizontal surface. This causes a tip shape to form. Further down, the wire is protected as the tungstate $\left(\mathrm{WO}_{4}{ }^{2-}\right)$ ions fall along the sides of the wire, forming an increasingly dense laminar layer that protects the lower end of the tip from being etched. When the diameter at the meniscus is sufficiently decreased, gravity will break off the lower part of the wire.

Temper-annealed, 250- $\mu \mathrm{m}$-diameter, $99.95 \%$ purity polycrystalline tungsten wire was used in the fabrication. The electrolyte was made by dissolving potassium hydroxide $(\mathrm{KOH})$ in deionised water, producing a $7.5 \mathrm{~mol}$ $\mathrm{L}^{-1}$ concentration aqueous solution. The second electrode used was a tinned copper electrical wire with a diameter of $0.3 \mathrm{~mm}$. The tungsten wire was pre-etched for five seconds at $4 \mathrm{~V}$ to reduce the surface roughness. After pre-etching, the wire was lifted $\sim 1 \mathrm{~mm}$ before continuing the etching process at $4 \mathrm{~V}$ until the lower part fell off. The total etching time was approximately two minutes.

\section{Experimental setup}

A fibre-coupled external cavity diode laser at $1.55 \mu \mathrm{m}$, connected to an erbium-doped fibre amplifier, was used as the light source in the experiments. To obtain spectral measurements of the microresonator mode, the frequency of the laser source was scanned by current modulation with a triangular wave signal at $1007 \mathrm{~Hz}$ (Fig. 3) and $20 \mathrm{~Hz}$ (Fig. 4). As the light was subsequently fed into an amplifier operating at saturation, the optical power was kept constant. A polarisation controller was used for optimising the coupling to a resonator mode, and a circulator was used to separate the backward-propagating light in the tapered fibre for detection. The tapered fibre was mounted on a manual piezo stage to control the coupling to the resonator. Amplified photodetectors and an oscilloscope were used for simultaneously monitoring the transmitted and backscattered light.

The tungsten tip was fixed to a polylactic acid (PLA) plastic mount sitting on a three-axis piezo positioner. The tip position was raster scanned over a $1 \mu \mathrm{m} \times 1.5 \mu \mathrm{m}$ area in the resonator plane, and backscattering and transmission spectra were obtained for each $50 \mathrm{~nm}$ (Fig. 3) or $25 \mathrm{~nm}$ (Fig. 4) step. The tip positioner and oscilloscope were simultaneously computer controlled, allowing a capture time of a few minutes for each of the two measurements reported.

\section{Data analysis}

Least squares fitting procedures were applied to the spectra obtained in the measurement to determine the pump transmission half-linewidth $\gamma$ and backscattering amplitude $A_{\mathrm{b}}$ of the cavity for each position in the measurement. No spectrally resolvable mode splitting was observed.

For the pump resonance, a normal Lorentzian dip from a background $B$ was used, $B-A_{\mathrm{p}} /\left(1+\delta^{2} / \gamma^{2}\right)$, where the detuning with respect to the resonance angular frequency $\omega_{0}$ is $\delta=\omega-\omega_{0}$. However, the spectral shape for the backscattering is distorted, as it is effectively pumped by a Lorentzian pulse (the pump resonance), resulting in a lineshape of $A_{\mathrm{b}} /\left(1+\delta^{2} / \gamma^{2}\right)^{2}$. in the small backscattering limit (see Supplementary information for the derivation).

Subsequent to fitting the individual measurements, the grid data of the linewidth and backscattering amplitude measurements were fitted. The functions used for the grid data fitting are expressed in a rotated (Cartesian) coordinate system $(r, \phi)$ at an angle $\beta$ to the measurement coordinate system $(x, y)$, where the coordinate transformation is given by

$$
\left(\begin{array}{l}
r \\
\phi
\end{array}\right)=\left(\begin{array}{cc}
\cos \beta & -\sin \beta \\
\sin \beta & \cos \beta
\end{array}\right)\left(\begin{array}{l}
x \\
y
\end{array}\right)
$$


In this coordinate system, the $r$ axis is normal to the resonator surface and can be approximated as the azimuthal position over a short distance compared to the resonator radius of curvature. The rotation angle $\beta$ was determined as one of the free parameters of the linewidth grid fit, where the linewidth function expressed in the $(r, \phi)$ coordinate system is

$$
\gamma(r, \phi)=\gamma_{0}+\left\{\begin{array}{cc}
a_{\mathrm{p}}+s\left(r-r_{0}\right) & \text { for } r-r_{0}<0 \\
a_{\mathrm{p}} e^{-2 \alpha_{\gamma}\left(r-r_{0}\right)} & \text { for } r-r_{0} \geq 0
\end{array}\right.
$$

with the unperturbed linewidth $\gamma_{0}$, decay coefficient $\alpha_{\gamma}$, amplitude of the exponential decay $a_{\mathrm{p}}$, linear slope of the plateau at the resonator surface $s$, and coordinate system offset $r_{0}$ as free parameters.

The relative backscattering amplitude grid was subsequently fitted with the parameters $\beta$ and $r_{0}$ fixed to the values obtained from the linewidth fit. Only the portion of data outside the resonator boundary, $r-r_{0}=R \geq 0$, was fitted. The function fitted for the backscattering amplitude $A_{\mathrm{b}}$ is derived in the Supplementary information and reads

$$
A_{\mathrm{b}}=\left\{\begin{array}{cc}
\text { not fitted } & \text { for } R<0 \\
|g|^{2} / \gamma^{4}(r, \phi) & \text { for } R \geq 0
\end{array}\right.
$$

where $\gamma(r, \phi)$ is the fitted linewidth function, and the coupling from the forward-propagating to the backpropagating mode is

$$
|g|^{2}=g_{0}^{2}+2 g_{0} a_{\mathrm{t}} e^{-2 \alpha_{\mathrm{b}} R} \cos (\Theta)+a_{\mathrm{t}}^{2} e^{-4 \alpha_{\mathrm{b}} R}
$$

where $\gamma(r, \phi)$ is the fitted linewidth function, $g_{0}$ is the intrinsic backscattering strength, and $\Theta=k_{\mathrm{fr}} \phi+\theta+\theta_{R} R$ is a position-dependent phase responsible for the fringe pattern, in which $\theta_{R}$ is a radially dependent phase accounting for the shape of the tip and/or drift. The period $\Delta$ of the fringe pattern is given by $k_{\mathrm{fr}}=2 \pi / \Delta$.

To sample arbitrary lines in the two-dimensional grid of measurement data shown in Figs. 3c-e and 4b, c, the grid data were linearly interpolated.

\section{Acknowledgements \\ This work has been supported by the National Physical Laboratory Strategic Research Programme and the European Research Council (CounterLight, 756966), Marie Skłodowska-Curie Actions (MSCA) (CoLiDR, 748519) and UK Research and Innovation (MR/S032924/1). A.Ø.S. is supported by an Aker Scholarship and the Engineering and Physical Sciences Research Council (EPSRC) via the Quantum Systems Engineering programme at Imperial College London. J.M.S. acknowledges funding via a Royal Society of Engineering fellowship. L.D.B. and M.T.M.W. are supported by the EPSRC through the Centre for Doctoral Training in Applied Photonics. S.Z. acknowledges funding via an MSCA fellowship (GA-2015-713694).}

\section{Author details}

${ }^{1}$ National Physical Laboratory, Teddington TW11 OLW, UK. ${ }^{2}$ Blackett Laboratory, Imperial College London, London SW7 2AZ, UK. ${ }^{3}$ Clarendon Laboratory, University of Oxford, Oxford OX1 3PU, UK. ${ }^{4}$ City University of London, London
EC1V OHB, UK. ${ }^{5}$ Max Planck Institute for the Science of Light, Staudtstaße 2, 91058 Erlangen, Germany. ${ }^{6}$ Heriot-Watt University, Edinburgh EH14 4AS, UK.

${ }^{7}$ Friedrich Alexander University Erlangen-Nuremberg, 91058 Erlangen, Germany

\section{Author contributions}

A.Ø.S. and P.D'H. conceived the experiments. A.Ø.S., L.D.B., J.M.S., S.Z. and M.T. M.W. designed and constructed the experiments. A.Ø.S. performed the experiments and analysed the results with J.M.S. All authors discussed the project and its results and contributed to the manuscript.

Funding

Open Access funding enabled and organized by Projekt DEAL.

\section{Data availability}

The data that support this article are available from the corresponding author upon reasonable request.

\section{Code availability}

The code for data analysis that supports the plots within this article is available from the corresponding author upon reasonable request.

\section{Conflict of interest}

The authors declare that they have no conflict of interest.

Supplementary information is available for this paper at https://doi.org/ 10.1038/s41377-020-00440-2.

Received: 3 April 2020 Revised: 3 November 2020 Accepted: 19 November 2020

Published online: 23 December 2020

\section{References}

1. Heylman, K. D. et al. Optical microresonators for sensing and transduction: a materials perspective. Adv. Mater. 29, 1700037 (2017).

2. Foreman, M. R., Swaim, J. D. \& Vollmer, F. Whispering gallery mode sensors. Adv. Opt. Photon. 7, 168-240 (2015).

3. Righini, G. C. et al. Whispering gallery mode microresonators: fundamentals and applications. La Riv. del. Nuovo Cim. 34, 435-488 (2011).

4. Kippenberg, T. J., Holzwarth, R. \& Diddams, S. A. Microresonator-based optical frequency combs. Science 332, 555-560 (2011).

5. Matsko, A. B. et al. Whispering-gallery-mode resonators as frequency references. I. Fundamental limitations. J. Optical Soc. Am. B 24, 1324-1335 (2007).

6. Del'Haye, P. et al. Optical frequency comb generation from a monolithic microresonator. Nature 450, 1214-1217 (2007).

7. Aspelmeyer, M., Kippenberg, T. J. \& Marquardt, F. Cavity optomechanics. Rev. Mod. Phys. 86, 1391-1452 (2014).

8. Wilson, D. J. et al. Measurement-based control of a mechanical oscillator at its thermal decoherence rate. Nature 524, 325-329 (2015).

9. Enzian, G. et al. Observation of Brillouin optomechanical strong coupling with an $11 \mathrm{GHz}$ mechanical mode. Optica 6, 7-14 (2019).

10. Strekalov, D. V. et al. Nonlinear and quantum optics with whispering gallery resonators. J. Opt. 18, 123002 (2016).

11. Fürst, J. U. et al. Quantum light from a whispering-gallery-mode disk resonator. Phys. Rev. Lett. 106, 113901 (2011).

12. Marin-Palomo, P. et al. Microresonator-based solitons for massively parallel coherent optical communications. Nature 546, 274-279 (2017).

13. Reimer, $C$. et al. Generation of multiphoton entangled quantum states by means of integrated frequency combs. Science 351, 1176-1180 (2016).

14. Pfeifle, J. et al. Coherent terabit communications with microresonator Kerr frequency combs. Nat. Photon. 8, 375-380 (2014).

15. Mohageg, M., Savchenkov, A. \& Maleki, L. Coherent backscattering in lithium niobate whispering-gallery-mode resonators. Opt. Lett. 32, 2574-2576 (2007).

16. Kippenberg, T. J., Spillane, S. M. \& Vahala, K. J. Modal coupling in traveling-wave resonators. Opt. Lett. 27, 1669-1671 (2002).

17. Gorodetsky, M. L., Pryamikov, A. D. \& Ilchenko, V. S. Rayleigh scattering in highQ microspheres. J. Optical Soc. Am. B 17, 1051-1057 (2000). 
18. Weiss, D. S. et al. Splitting of high-Q Mie modes induced by light backscattering in silica microspheres. Opt. Lett. 20, 1835-1837 (1995).

19. Chow, W. W. et al. The ring laser gyro. Rev. Mod. Phys. 57, 61-104 (1985).

20. Dell'Olio, F. et al. Recent advances in miniaturized optical gyroscopes. J. Eur. Optical Soc. Rapid Publ. 9, 14013 (2014).

21. Liang, W. et al. Resonant microphotonic gyroscope. Optica 4, 114-117 (2017)

22. Yang, Q. F. et al. Counter-propagating solitons in microresonators. Nat. Photon. 11, 560-564 (2017).

23. Lucas, E. et al. Spatial multiplexing of soliton microcombs. Nat. Photon. 12 , 699-705 (2018).

24. Del Bino, L. et al. Symmetry breaking of counter-propagating light in a nonlinear resonator. Sci. Rep. 7, 43142 (2017).

25. Kaplan, A. E. \& Meystre, P. Enhancement of the Sagnac effect due to nonlinearly induced nonreciprocity. Opt. Lett. 6, 590-592 (1981).

26. Moroney, N. et al. Logic gates based on interaction of counterpropagating light in microresonators. J. Lightwave Technol. 38, 1414-1419 (2020).

27. Del Bino, L., Moroney, N. \& Del'Haye, P. Optical memories and switching dynamics of counterpropagating light states in microresonators. Preprint at arXiv 2002.02954 (2020).

28. Del Bino, L. et al. Microresonator isolators and circulators based on the intrinsic nonreciprocity of the Kerr effect. Optica 5, 279-282 (2018).

29. Kim, E., Baaske, M. D. \& Vollmer, F. Towards next-generation label-free biosensors: recent advances in whispering gallery mode sensors. Lab Chip 17, 1190-1205 (2017).

30. Little, B. E. et al. Microring resonator arrays for VLSI photonics. IEEE Photon. Technol. Lett. 12, 323-325 (2000).

31. Matres, J. \& Sorin, W. V. Simple model for ring resonators backscatter. Opt. Express 25, 3242-3251 (2017).

32. MacKintosh, F. C. \& John, S. Coherent backscattering of light in the presence of time-reversal-noninvariant and parity-nonconserving media. Phys. Rev. B 37, 1884-1897 (1988)
33. Golubentsev, A. A. Suppression of interference effects in multiple scattering of light. Sov. Phys. JETP Engl. Transl. 59, 26-32 (1984).

34. Wiersig, J. Structure of whispering-gallery modes in optical microdisks perturbed by nanoparticles. Phys. Rev. A 84, 063828 (2011).

35. Mazzei, A. et al. Controlled coupling of counterpropagating whispering-gallery modes by a single Rayleigh scatterer: a classical problem in a quantum optical light. Phys. Rev. Lett. 99, 173603 (2007).

36. Zhu, J. G. et al. Controlled manipulation of mode splitting in an optical microcavity by two Rayleigh scatterers. Opt. Express 18, 23535-23543 (2010).

37. Peng, B. et al. Chiral modes and directional lasing at exceptional points. Proc Natl Acad. Sci. USA 113, 6845-6850 (2016).

38. Wang, C. Q. et al. Electromagnetically induced transparency at a chira exceptional point. Nat. Phys. 16, 334-340 (2020).

39. Kim, S., Taylor, J. M. \& Bahl, G. Dynamic suppression of Rayleigh backscattering in dielectric resonators. Optica 6, 1016-1022 (2019).

40. Spillane, S. M. et al. Ideality in a fiber-taper-coupled microresonator system for application to cavity quantum electrodynamics. Phys. Rev. Lett. 91, 043902 (2003).

41. Zhu, J. G. et al. Nonlinear characterization of silica and chalcogenide microresonators. Optica 6, 716-722 (2019).

42. De Fornel, F. Evanescent Waves (Berlin Heidelberg: Springer-Verlag, 2001)

43. Götzinger, S., Benson, O. \& Sandoghdar, V. Influence of a sharp fiber tip on high-Q modes of a microsphere resonator. Opt. Lett. 27, 80-82 (2002).

44. Del'Haye, P., Diddams, S. A. \& Papp, S. B. Laser-machined ultra-high-Q microrod resonators for nonlinear optics. Appl. Phys. Lett. 102, 221119 (2013).

45. Ibe, J. P. et al. On the electrochemical etching of tips for scanning tunneling microscopy. J. Vac. Sci. Technol. A 8, 3570-3575 (1990).

46. Hagedorn, $\mathrm{T}$. et al. Refined tip preparation by electrochemical etching and ultrahigh vacuum treatment to obtain atomically sharp tips for scanning tunneling microscope and atomic force microscope. Rev. Sci. Instrum. 82 113903 (2011). 